Running Title: Time varying lambda and structures

Article type: Original Research

\title{
Time varying cosmological constant and its implications for structure formation
}

\section{Louise Rebecca $^{1}$, C. Sivaram ${ }^{2}$, Arun Kenath ${ }^{1,3}$}

\begin{abstract}
Although the presence of dark energy is well established from various observations, its true nature is still not well understood. The cosmological constant term seems to be the preferred candidate. In earlier work we had a constant cosmological constant term to limit the sizes of large-scale structures at lower redshifts. In this work, we extend this to large scale structures at higher redshifts. Here we invoke a time varying cosmological constant to set constraints on sizes of galaxies at high redshifts and see that they are consistent with their observed sizes. The time-varying cosmological constant also provides a possible solution to the puzzle of structure formation of large disk galaxies (like the Wolfe disk) observed at very early stages of the Universe. Future observations of galaxies at even higher redshifts could support our approach.
\end{abstract}

Keywords: Cosmological constant; dark energy; structure formation; early Universe

\footnotetext{
*Corresponding author

${ }^{1}$ Department of Physics, Christ Junior College, Bengaluru, 560029, Karnataka, India

${ }^{2}$ Indian Institute of Astrophysics, Bengaluru, 560034, Karnataka, India

${ }^{3}$ Department of Physics and Electronics, CHRIST (Deemed to be University), Bengaluru, 560029, Karnataka, India

E-mail addresses: louise.rheanna@cjc.christcollege.edu (Louise Rebecca); sivaram@iiap.res.in (C Sivaram); kenath.arun@cjc.christcollege.edu (Arun Kenath)
} 


\section{Introduction}

The energy density of the Universe as obtained from detailed cosmological observations accounts for around $30 \%$ of the critical density. This leaves the remaining $70 \%$ of the energy density unaccounted for (Perlmutter et al., 1999, Riess et al., 1998.). The missing energy density is also found to be associated with the accelerated expansion of the Universe. This accelerated expansion of the Universe is attributed to the so-called dark energy (DE) which forms a source of repulsive gravity. Though the presence of dark energy is well established its true nature still remains a mystery. There are various models of dark energy, some of which include the cosmological constant term, quintessence, phantom energy, quintom DE, Chaplygin gas, etc. The cosmological constant term is found to be the most consistent with current observations.

The cosmological constant is the energy density of vacuum, originally introduced by Einstein as an addition to his theory of general relativity (GR) to make the Universe static (Einstein, 1917). If the Universe is filled with just ordinary matter and radiation, GR predicts that the gravitational attraction of all matter in the Universe would pull it back and slow down the rate of expansion, i.e., decelerate it. Einstein's field equation thus included a cosmological constant term to counteract the gravitational attraction of all the matter in the Universe, and hence make the Universe static, following the equation:

$R_{\mu \nu}-\frac{1}{2} g_{\mu \nu} R+g_{\mu \nu} \Lambda=\frac{8 \pi G}{c^{4}} T_{\mu \nu}$

where, $\Lambda$ is a negative pressure term called the cosmological constant that causes repulsive gravitation.

\section{Time-varying cosmological constant}

The cosmological constant term need not be a constant, and could evolve with time. In an earlier work (Sivaram et al., 2020a), we considered a cosmological constant that varies with time as,

$\Lambda=\Lambda_{0}\left(\frac{t_{0}}{t}\right)^{2}$

where $t_{0}$ is taken as Planck time, i.e., $t_{0}=t_{P l} \approx 10^{-44} s$ and $\Lambda_{0}=\Lambda_{P l}$ is the value of $\Lambda$ at Planck time. Such a time variation was first considered, as early as 1976 (Sinha et al., 1976) giving an inflationary picture with a large initial value of $\Lambda$ (quantum vacuum energy).

At the Planck epoch, the cosmological constant and the dark energy density respectively are, 
$\Lambda_{P l}=\Lambda_{0}=\left(\frac{c^{3}}{\hbar G}\right) \approx 10^{66} \mathrm{~cm}^{-2}$

and

$\rho_{\Lambda_{0}}=\frac{\Lambda_{0} c^{2}}{8 \pi G} \approx 10^{93} \mathrm{gcm}^{-3}$

From equation (2), the cosmological constant and hence the dark energy density for the present epoch are,

$\Lambda=\Lambda_{0}\left(\frac{t_{0}}{t}\right)^{2} \approx 10^{-56} \mathrm{~cm}^{-2}$

and

$\rho=\rho_{0}\left(\frac{t_{0}}{t}\right)^{2} \approx 10^{-29} \mathrm{gcm}^{-3}$

which are the same as that observed at the present epoch (Ade et al., 2016).

\section{Dark energy constraints on structure formation}

A recent study spotted the oldest disk-shaped galaxy DLA0817g (also named as the Wolfe Disk) formed just 1.5 billion years after the Big Bang (Neeleman et al., 2020). Most disk-shaped galaxies like the Milky Way formed gradually and reached their sizes at relatively later epochs. The discovery of a massive rotating disk-shaped galaxy with properties similar to the Milky Way provides challenges to traditional models of structure formation. This puzzle can be solved by a time-varying cosmological constant.

As established in earlier works (Rebecca et al., 2018; 2020; Sivaram et al., 2020b), for large scale structures it is required that their gravitational binding self-energy density must at least equal the background repulsive dark energy density, i.e.:

$\frac{G M^{2}}{8 \pi R^{4}}=\frac{\Lambda c^{4}}{8 \pi G}$

This implies a mass-radius relation of,

$\frac{M}{R^{2}} \approx \frac{c^{2}}{G} \sqrt{\Lambda}$

If the dark energy is a constant $\left(\Lambda \approx 10^{-56} \mathrm{~cm}^{-2}\right)$, the value of $\frac{M}{R^{2}}$ turns out to be $1 \mathrm{~g} / \mathrm{cm}^{2}$. This holds good for vast range of structures from globular clusters to galaxies as well as galaxy clusters up to the Hubble volume (Sivaram et al., 2013). It was also shown that the ratio $\left(M / R^{2}\right)$ for various galaxies, from the early primeval galaxy, to low $\mathrm{z}$ galaxies (in the present epoch) are in agreement with the constraint set by equations (7) to (8) (Rebecca et al., 2018). 
The time-varying cosmological constant that follows equation (2) for the Wolfe Disk galaxy (at $t \approx 4.7 \times 10^{16} \mathrm{~s}$ ) turns out to be $\Lambda \approx 10^{-53} \mathrm{~cm}^{-2}$. This, from equation (8), then implies that the ratio $M / R^{2}$ will be larger (since $\Lambda$ is larger) at that epoch. Hence facilitating the formation of massive structures with more compact sizes. This then provides a possible resolution to the concern of formation of such large galaxies at such early epochs.

In earlier works (Sivaram et al, 2013; 2012) it was also pointed out that for large scale structures such as galaxies, galaxy clusters and superclusters the repulsive cosmological dark energy can limit the sizes to which such clusters can grow, i.e., to the point where the attractive gravitational force matches the repulsive dark energy density. For a gravitationally bound structure, we have:

$\frac{G M}{R}-\frac{1}{3} \Lambda c^{2} R^{2} \geq 0$

where, $\frac{G M}{R}$ is the gravitational potential and $\frac{1}{3} \Lambda c^{2} R^{2}$ is the potential due to dark energy. Equation (9) implies constraints on the sizes of large gravitationally bound structures such as galaxy clusters and superclusters. Their size is limited as:

$R_{\text {lim }} \leq\left(\frac{3 G M}{\Lambda c^{2}}\right)^{1 / 3}$

Table 1 makes a comparison of the size constraint obtained from equation (9) for some of the oldest structures with a constant cosmological constant term to that with a time-varying cosmological term that follows equation (2). As can be seen, a time varying cosmological constant is more consistent with the observed size limit than that with the constant value of $\Lambda$.

\begin{tabular}{|c|c|c|c|c|c|}
\hline Galaxy & Redshift (z) & $\begin{array}{c}\Lambda(\text { at the } \\
\text { corresponding } z) \\
\left(\text { in } \mathrm{cm}^{-2}\right)\end{array}$ & $\begin{array}{c}\boldsymbol{R}_{\text {observed }} \\
\text { (in cm) }\end{array}$ & $\begin{array}{l}R_{\text {lim }}^{\text {const } \Lambda} \\
(\text { in } \mathrm{cm})\end{array}$ & $\begin{array}{l}R_{\text {lim }}^{\text {vary } \Lambda} \\
(\text { in } \mathrm{cm})\end{array}$ \\
\hline$G N-z 11$ & 11.09 & $10^{-52}$ & $3.7 \times 10^{21}$ & $3.05 \times 10^{23}$ & $1.6 \times 10^{22}$ \\
\hline SPT0615-JD & 9.9 & $10^{-53}$ & $2.3 \times 10^{21}$ & $5 \times 10^{23}$ & $5 \times 10^{22}$ \\
\hline$E G S-z s 8-1$ & 9.1 & $10^{-53}$ & $9 \times 10^{21}$ & $8.5 \times 10^{23}$ & $8.6 \times 10^{22}$ \\
\hline$A 1689-z D 1$ & 7.5 & $10^{-53}$ & $1.4 \times 10^{21}$ & $4.2 \times 10^{23}$ & $4 \times 10^{22}$ \\
\hline GN-108036 & 7.213 & $10^{-53}$ & $2 \times 10^{21}$ & $7 \times 10^{23}$ & $7 \times 10^{22}$ \\
\hline
\end{tabular}

Here, $R_{\text {lim }}^{\text {const } \Lambda}$ is the limiting size of the structure with constant value of $\Lambda \approx 10^{-56} \mathrm{~cm}^{-2}$, and $R_{\text {lim }}^{\text {vary } \Lambda}$ is the limiting size where the $\Lambda$ term is evolving over time according to equation (2). 


\section{Conclusion}

In an earlier work, a constant cosmological constant term $(\Lambda)$ was used to limit the sizes of large-scale structures at lower redshifts. Here we find that a time-dependent cosmological constant varying according to equation (2) gives constraints for the sizes of large-scale structures (galaxies, clusters, etc.) at high redshifts which are closer to their actually observed sizes. The time varying cosmological constant has been invoked in our earlier works in a different context (early universe inflation). Future observations of galaxies at even higher redshifts, for instance from JWST, could support our approach. With the time-varying cosmological constant we also attempt to give a possible solution to the puzzle of structure formation for large disk galaxies like the Wolfe disk observed at very early stages of the Universe.

\section{References}

- Ade, P.A.R., et al.: Planck 2015 results XIII. Cosmological parameters. Astron. Astrophys. 594, A13 (2016)

- Einstein, A.: Cosmological considerations in the general theory of relativity. Sitz. Konig. Preuss. Akad. part 1, 142 (1917)

- Neeleman, M., et al.: A cold, massive, rotating disk galaxy 1.5 billion years after the big bang. Nature. 581, 269 (2020)

- Perlmutter, S., et al.: Measurements of omega and lambda from 42 high-redshift supernovae. Astrophys. J., 517, 565 (1999)

- Rebecca, L., Arun, K., Sivaram, C.: Dark energy constraints on masses and sizes of large scale cosmic structures. Astrophys. Space Sci. 363, 149 (2018)

- Rebecca, L., Arun, K., Sivaram, C.: Dark matter density distributions and dark energy constraints on structure formation including MOND, Indian J. Phys., 94, 1491 (2020)

- Riess, G., et al.: Observational evidence from supernovae for an accelerating universe and a cosmological constant. Astron. J. 116, 1009 (1998)

- Sinha, K.P., Sivaram, C., Sudarshan, E.C.G.: The superfluid vacuum state, time-varying cosmological constant, and nonsingular cosmological models. Found. Phys. 6, 717 (1976)

- Sivaram, C., Arun, K.: Primordial rotation of the Universe, hydrodynamics, vortices and angular momenta of celestial objects. Open Astron. J. 5, 7 (2012)

- Sivaram, C., Arun, K.: Holography, dark energy and entropy of large cosmic structures. Astrophys. Space Sci. 348, 2 (2013) 
- Sivaram, C., Arun, K., Rebecca, L.: Planckian pre big bang phase of the Universe. Astrophys. Space Sci. 365, 17 (2020a)

- Sivaram, C., Arun, K., Rebecca, L.: MOND, MONG, MORG as alternatives to dark matter and dark energy, and consequences for cosmic structures, J. Astrophys. Astron., 41, 4 (2020b) 